\title{
Necrotizing fasciitis of the jaw, neck and mediastinum caused by Klebsiella oxytoca and Streptococcus constellatus: a case report
}

\author{
Ling Jin", Kai Fan", Shuangxi Liu, Shaoqing Yu \\ Department of Otolaryngology, Head and Neck Surgery, Tongji Hospital of Tongji University, Tongji University School of Medicine, Shanghai, \\ China \\ \#These authors contributed equally to this work. \\ Correspondence to: Shaoqing Yu. Department of Otolaryngology, Tongii Hospital, 389 Xincun Road, Putuo District, Shanghai 200065, China. \\ Email: yu_shaoqing@163.com.
}

\begin{abstract}
Necrotizing fasciitis (NF) is a rare, fulminant, lethal soft-tissue infection result in fascial necrosis, it is rarer in the head and neck area. Infection caused by Klebsiella oxytoca is much less common. Therefore, we reported a case of NF in the maxillofacial region, neck and upper mediastinum caused by Klebsiella oxytoca as the main cause recently treated in our department. The patient is a middle-aged male with a 10 -year history of diabetes with unstable insulin control. The main symptoms were pain on left side with dysphagia and fever and the situation was getting worse. The patient had limited ability to have mouth open and had hyperemia, swelling on the left pharynx, maxillofacial area, and upper left neck and skin tenderness, and all symptoms were getting worse quick. The CT mainly found out that left oropharyngeal wall, parotid gland area, bilateral submaxilla, left neck, and superior mediastinum are swelling with gas. The blood test result: leukocytes count $16.64 \times 10^{9} / \mathrm{L}$, neutrophils percentage $85.8 \%$; C-Pr $320 \mathrm{mg} / \mathrm{L}$; urinary routine: urine glucose (+++++), ketone bodies (+++++); fasting glucose metabolism: glucose $21.33 \mathrm{mmol} / \mathrm{L}$, glycosylated albumin $47.67 \%$. Three incisions of facial and neck were performed to drain pus. Result of bacteria culture: Klebsiella oxytoca and Streptococcus constellatus During treatment of DKA and reduce patient glucose level, we also treated patient with neck and trachea incisions to drain pus and cleaned daily wound area and used different antibiotics according to the bacteria culture and CT results. And finally, the patient was cured and discharged from hospital. This case of NF was very rare not only the bacteria in this case but also the pathological changes related (involving the mediastinum). The report of his diagnosis and treatment can provide experience for future treatments.
\end{abstract}

Keywords: Necrotizing fasciitis; Klebsiella oxytoca; Streptococcus constellatus; diabetes mellitus

Submitted Dec 06, 2020. Accepted for publication Mar 18, 2021.

doi: 10.21037/apm-20-2427

View this article at: http://dx.doi.org/10.21037/apm-20-2427

\section{Introduction}

Necrotizing fasciitis (NF) is a rare, life-threatening infectious disease characterized by deep and superficial fascial necrosis, with rapid onset and rapid progression. It generally occurs in the perineum, groin and extremities, rarely in the head and neck (1). The complications of NF in the neck are serious and the mortality rate is relatively high (2). Hemolytic Streptococcus A, Staphylococcus spp. is the most common causative agent of NF. However, we reported a recently successfully treated case of severe NF that occurs around the face, neck, and mediastinum which is caused by rare pathogens (Klebsiella oxytoca and Streptococcus constellatus). The case will help us to have more clinic experience and create early-stage diagnostic standard and anticipate the severance of the case. And it will allow us to choose the correct treatment on this disease and other complication associated.

We present the following article in accordance with the CARE reporting checklist (available at http://dx.doi. 

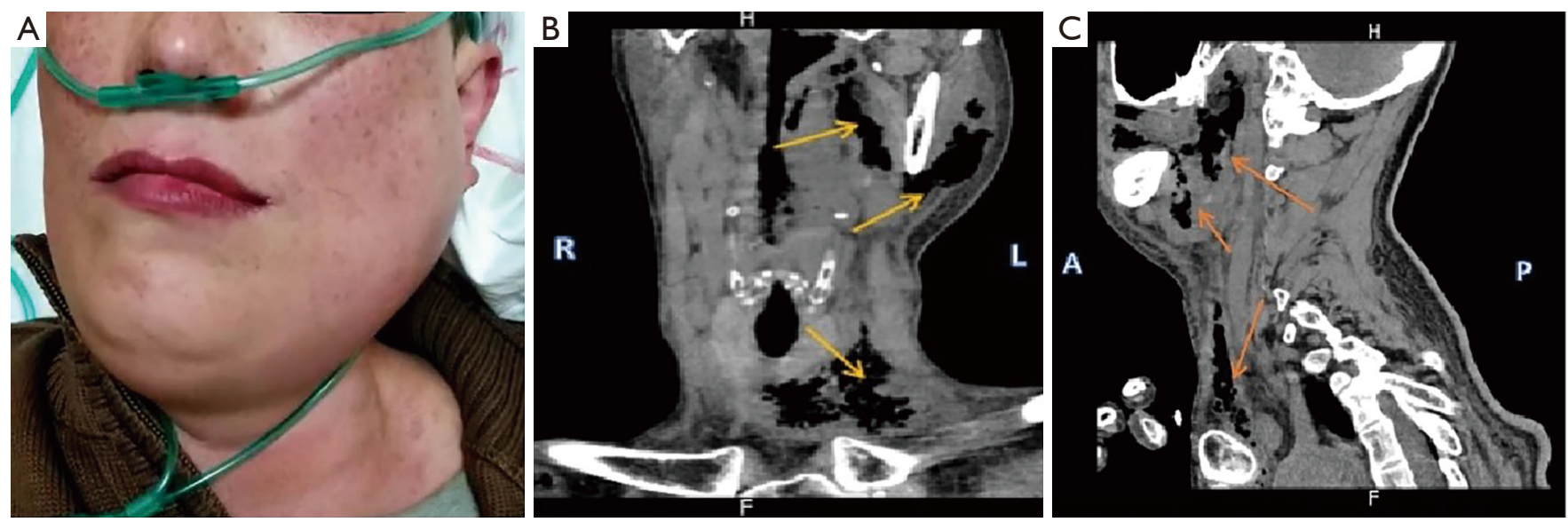

Figure 1 Preoperative frontal view, preoperative coronary CT (computed tomography) and preoperative sagittal CT. (A) Preoperative frontal view: significant swelling in the left parotid area, submandibular, upper neck. (B) Preoperative coronary CT: left parotid area, submandibular, lower neck air-containing pus cavity (arrow). (C) Preoperative sagittal CT: left fundus, submandibular, upper mediastinal airbearing.

org/10.21037/apm-20-2427).

\section{Case presentation}

The patient, male, 48 years old, was admitted to the department of ENT on 17 March 2020 with symptoms "4-days sore in left throat: and half day dysphagia and fever". He had experienced cold and fatigue without toothache. Patient had also 10 years diabetes history with unstable control with insulin. During his check in patient had below condition: temperature: $38.5^{\circ} \mathrm{C}$, heart rate: $108 \mathrm{bpm}$, blood pressure: $130 / 80 \mathrm{mmHg}$, breathing 20 beats/min. Clear consciousness, restricted mouth open ability (1 transverse finger); Lower left side with no teeth or only roots left; $2^{\text {nd }}$ degree swelling on left side tonsil; Obvious swelling of the soft palate and palatal tongue arch on the left side, inaccessible to observe condition of epiglottis, larynx and pharynx; congestion of the skin in the parotid area, submaxillary area and upper left neck mild swelling with tenderness. The blood test result: leukocytes count $16.64 \times 10 \%$ L, neutrophils percentage $85.8 \%$; C-Pr $320 \mathrm{mg} / \mathrm{L}$; urinary routine: urine glucose $(+++++)$, ketone bodies $(+++++)$; fasting glucose metabolism: glucose $21.33 \mathrm{mmol} / \mathrm{L}$, glycosylated albumin $47.67 \%$. Normal liver and kidney function, negative screening for human immunodeficiency virus (HIV) and syphilis. Originally was considered as peritonsillar abscess with neck space infection disease. Puncture was performed to extract pus, but no pus was extracted. Cefathiamidine, metronidazole and insulin were injected to reduce blood glucose level and patient was rehydrated for ketoacidosis.

On the second day of admission, patient's symptoms worsened, he was apathetic, sleepy with heart rate of 110/min. Patient had difficulty to breath and swelling was getting worse on the left parotid area, submandibular and upper neck, skin temperature increased, and was feeling twisting (Figure 1A). Patient was provided oxygen inhalation and was under ECG monitoring. CT scan was performed to check head and neck areas including left oropharyngeal lateral wall, left facial parotid area, both sides of submandibular, left neck and upper mediastinal pneumoperitoneum (Figure 1B,C). Patient also had ketoacidosis symptom (blood analysis results: $\mathrm{PH} 7.31$, $\mathrm{CO} 2$ partial pressure $20 \mathrm{mmHg}$, oxygen partial pressure $77 \mathrm{mmHg}$, bicarbonate concentration $10.1 \mathrm{mmol} / \mathrm{L}$, standard bicarbonate $13.9 \mathrm{mmol} / \mathrm{L}$ ), change of sepsis (procalcitonin $8.73 \mathrm{ng} / \mathrm{mL}$, reference value $0.5-10 \mathrm{ng} / \mathrm{mL}$ for consideration of sepsis). During that night tracheotomy, neck incision and drainage were performed. During the surgery, a transverse incision was made under the right jaw, and taupe pus with smell was extracted from the area of subhyoid muscles. There was smell but no obvious pus observed from left anterior edge of sternocleidomastoid muscle to the peri-carotid artery sheath area, until the upper level of the tip of the mastoid process and lower level of the posterior area of the clavicular head. During the operation, the two incisions were connected in the subcutaneous space. 2 negative pressure drainage tubes and nasogastric feeding 

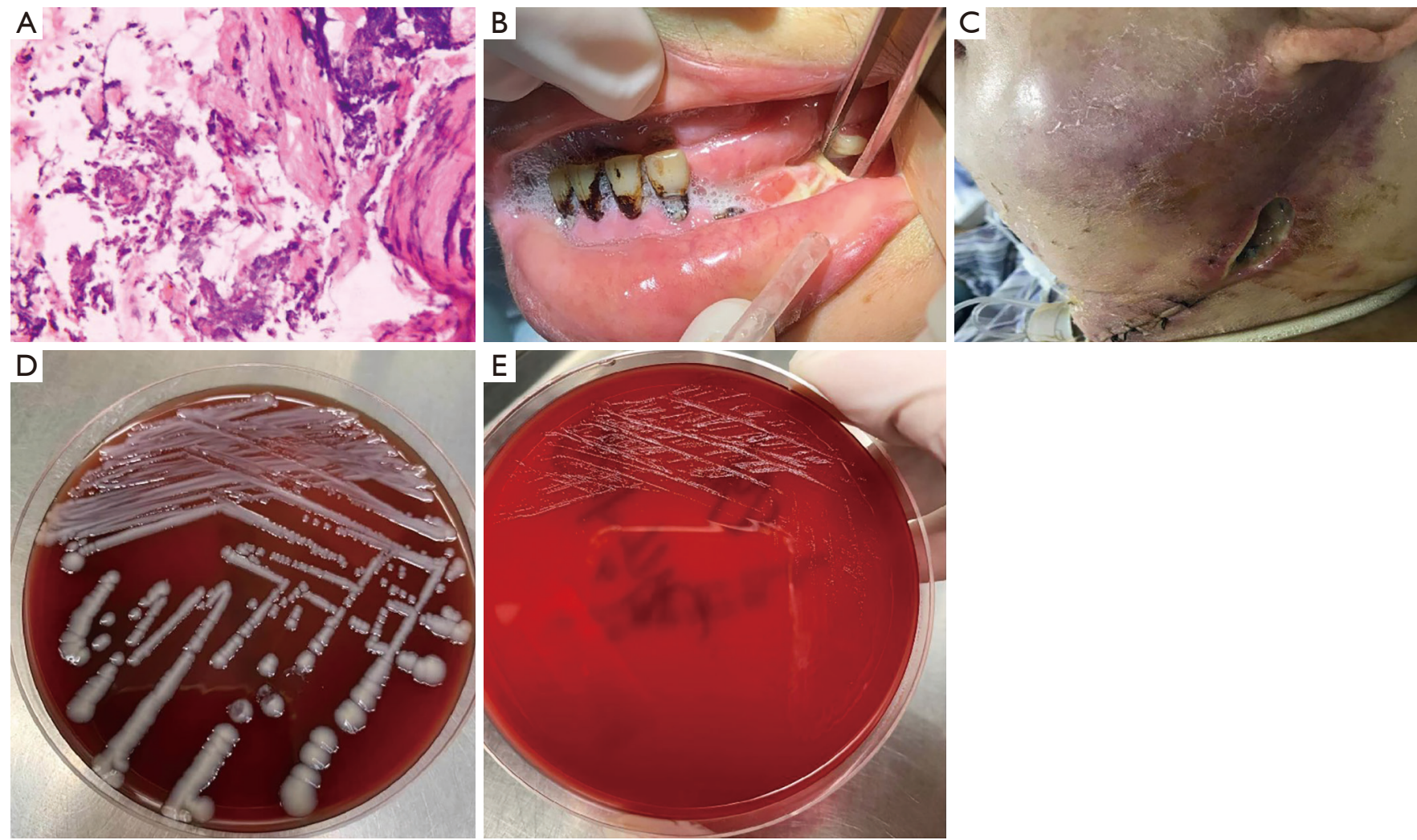

Figure 2 Pathological sections, clinical features and pictures of pathogenic bacteria of the patient. (A) Pathology: HE (hematoxylin-eosin staining, HE) $\times 200$, fascial necrosis and massive inflammatory cell infiltration. (B) Pus cavity rupture via the left sublingual caruncle. (C) After the second postoperative trauma: dark red, swollen skin in the left parotid area, open drainage port. (D) Klebsiella oxytoca: growth on blood plate, forming large greyish-white, moist, mucus-like colonies. (E) Streptococcus constellatus: white, round, $\beta$-hemolytic ring colonies on blood plates incubated at $35^{\circ} \mathrm{C}$.

tubes were inserted before patient was sending to SICU for monitoring

Next day after surgery, patient's temperature reached $40{ }^{\circ} \mathrm{C}$, and the swelling of the left side of the jaw and face was still obvious. Re-examination of the chest CT shows no improvement of gas build on the upper mediastinum and the left parotid area. So we rechecked through incision on the left neck, and a large amount of taupe pus with smell was drained from the anterior and subcutaneous space of the parotid gland. Pseudomembranous necrosis of the anterior muscular fascia was also observed, after cleaning the extracted tissue was sent for pathology check (Figure 2A); when we check the left side of mouth, mouth open ability was still limited, bottom of tongue was still swelling, ulceration and pus discharge at the left sublingual caruncle (Figure 2B); deep abscess was observed and connected to left parotid space neck space. Same type of pus was drained from left subcutaneous of left tracheotomy incision and upper mediastinum. After we left neck incision upper area open (Figure 2C); and drained pus under negative pressure from left bottom of mouth left neck incision. And clean abscess with hydrogen peroxide and diluted polyvinyl iodide solution.

Two pus cultures were sent for examination. The first pus culture sample was collected during the first surgery. The second pus culture sample was collected during the second surgery with trauma probe. The two pus cultures were both positive for Klebsiella oxytoca (Figure 2D) and Streptococcus constellatus (Figure 2E). Anaerobic cultures were negative for both pus and high fever blood. One week after treatment, pus and blood cultures showed fungal infection (Candida albicans and Candida guilliermondii). Before the bacterial culture results, based on experience, vancomycin, piperacillin sulbactam and ornidazole were 
used together, then adjusted to different antibiotic: meropenem and tigecycline, and fluconazole was used for fungal treatment. Throughout the entire treatment, glucose level was monitored and controlled by using insulin. Patient was rehydrated for ketoacidosis, nasal feeding and albumin supplementation were also used to treat his hypoproteinemia.

9 days after his surgery, patient temperature returned to normal. And from the $14^{\text {th }}$ days of admission to hospital, neck and oral pus decreased, and his mouth open ability improved as well. On the $25^{\text {th }}$ day with the $3^{\text {rd }}$ cleaning of wound, left neck incision was sutured. Only one negative pressure drain tube was left at the left front parotid gland abscess. On the $30^{\text {th }}$ day, negative pressure drain was removed. And patient was discharged from hospital on day 37. And according to patient's follow up visits after 3 months, 6 months and one year there was no recurrence.

All procedures performed in the studies involving human being as participants were in accordance with the ethical standards of the institutional and/or national research committee(s) and with the Helsinki Declaration (as revised in 2013). Written informed consent was obtained from the patient families.

\section{Discussion}

$\mathrm{NF}$ is dangerous and rare disease with an incidence of $0.004 \%$ to $0.001 \%$ (3), but there is a trend of increasing incidence worldwide. In the NF case, we presented, the situation was very severe, NF occurred extensively between shallow and deep fascial stroma. While it is more rarely that the case also impacted the maxilla, neck and mediastinum. NF pathogens are mostly mixed bacterial infections $(4,5)$. Hemolytic Streptococcus A and Staphylococcus spp are the most common pathogens that cause NF. Recently, Klebsiella pneumoniae has been found as a cause of NF (6).

In this case, the mixed infection of Klebsiella oxytoca and Streptococcus constellatus constellation is rare. Klebsiella oxytoca is part of enterobacteriaceae family which is gram stain negative and facultative anaerobes. These bacteria can ferment glucose and produce acid and gas. Diabetic patient's high-level glucose served as raw material in this case. So the CT result showed in this case showed a large amount of cumulated gas. In recent years, Streptococcus constellatus as a part of Streptococcus milleri family has gradually became one of the important pathogens causing purulent infections in the body with a few reports (7). Although it is aerobic Gram-positive, the anaerobic environment can increase its ability to grow. And co-infected with other bacteria its virulence can increase significantly. The patient had several negative anaerobic cultures of pus and blood, but both bacteria have alibility to multiple quickly under anaerobic conditions. The patient clinical condition is very typical anaerobic bacteria infection, showing gas and smell. The presence of Candida in the blood and pus cultures one week after treatment, we consider that it is related with using large dose of different types of antibiotics, not the original pathogen.

NF occurs well in adults with immune deficiencies such as diabetes (8). Dentinogenic infections are the most common cause of cervical NF, followed by parapharyngeal interstitial and peritonsillar infections (9). In this case, the patient was a diabetic patient with unstable control and low immunity. His early symptom was one side infection around tonsils. His late symptom was severe abscess around parotid gland and bottom of the tongue. And ulcerated is presented from the sublingual caruncle. Although patient doesn't have tool extraction and toothache history, but left side teeth having cavities and missing teeth with only root. So we consider the cause of his NF infection is by teeth infection.

CT examination is essential in NF diagnosis and is one of the reliable methods of diagnosis and follow up (10). CT imaging of the neck NF includes: loss of fat shadow in in the interstices of the neck, thickness increasing of the cervical broad muscle, partial fractur of cervical broad muscle, fluid and gas accumulation in the deep cervical fascia and pneumoperitoneum. The typical sign of NF image is "Bubble sign" (11). Pathology is one of the standards for the diagnosis of NF, usually necrosis of the fascia and thrombosis of small vessels will be observed. In the case studied, the pathology conforms (Figure 2A).

Early-stage diagnosis of NF is very important, according to our experience, the following: (I) patients with low immunity such as unstable control of diabetes; (II) pharynx or dental infections are as inducement; (III) the disease progresses rapidly; (IV) neck swelling and pain, subcutaneous twirling feeling, apathetic; (V) in CT examination, there was swelling and effusion in fascial space, especially with "Bubble sign".

The basic principle of treating NF is to make timely and extensive incision, repeatedly and thoroughly clear the trauma, and establish an unobstructed drainage (12). Most patients with NF have diabetes or ketoacidosis situation. We need to monitor patient closely and treat all complications then have surgery as soon as possible. The incision around neck area is challenging as it should be large and deep 
enough to clear necrotic facial tissue. But neck tissue is complicated, it contains important structure as carotid artery, trachea, esophagus, nerves... Overtreatment can damage these structures and be fatal. Also, before effectivity of antibiotics, the condition could get worse. It may need extra 2 or more surgeries according to the patient's condition. In our case the patient worse condition was on day 3 after the surgery, further necrosis was presented. There was totally 3 times surgery to clean and drain It is also mentioned that after incision and drainage, combined with negative pressure suction, can strengthen the effect of cleaning and drainage.

In addition, the maintenance of airway patency is the top priority during the treatment of NF in the head and neck, and timely and necessary tracheotomy is an important part of the steps during treatment, which can prevent the occurrence of asphyxia. Early tracheotomy should be considered in patients with moderate to severe laryngeal obstruction and difficulty in opening the mouth; postoperative tissue swelling is aggravated by intraoperative pulling and injury to the cervical gap and upper mediastinal tissue, which can compress the airway, and prophylactic tracheotomy Pressure on airway can prevent pulling and injury to the cervical gap and upper mediastinal tissue and avoid aggravation of swelling condition. Preventive tracheotomy should also be performed before surgery. In this case, our patient is in a typical situation, so tracheotomy was performed before surgery.

According to relevant literature, although the mortality rate of NF is relatively high, but there is almost no recurrence of NF reported after cure, and the patient in this case also had no recurrence within 1 year of follow-up. Also, we considered that the patients will pay more attention to their heath after being saved.

In conclusion, NF at the head and neck area is rare and dangerous and is often with several bacterial infections. In this case it is much less common with two bacteria (Klebsiella acidophilus and Streptococcus zoster). Neck swelling and pain, subcutaneous crepitation, apathetic, are early-stage symptoms for diagnosis. Imaging, especially CT, is most helpful for diagnosis and evaluate treatment, and incisional examination of extensive fascial necrosis and pathology check after surgery are also accurate diagnosis standard. Early-stage diagnosis and positive treatment, keeping airway unobstructed, adjustment of antibiotics according to test result of culture and CT scan, debridement by stages, prevention and treatment of complications are the key therapy.

\section{Acknowledgments}

The authors thank Dr. Margaret Ferretti for editing the paper.

Funding: None.

\section{Footnote}

Reporting Checklist: The authors have completed the CARE reporting checklist. Available at http://dx.doi.org/10.21037/ apm-20-2427

Conflicts of Interest: All authors have completed the ICMJE uniform disclosure form (available at http://dx.doi. org/10.21037/apm-20-2427). The authors have no conflicts of interest to declare.

Ethical Statement: The authors are accountable for all aspects of the work in ensuring that questions related to the accuracy or integrity of any part of the work are appropriately investigated and resolved. All procedures performed in the studies involving human being as participants were in accordance with the ethical standards of the institutional and/or national research committee(s) and with the Helsinki Declaration (as revised in 2013). Written informed consent was obtained from the patient families.

Open Access Statement: This is an Open Access article distributed in accordance with the Creative Commons Attribution-NonCommercial-NoDerivs 4.0 International License (CC BY-NC-ND 4.0), which permits the noncommercial replication and distribution of the article with the strict proviso that no changes or edits are made and the original work is properly cited (including links to both the formal publication through the relevant DOI and the license). See: https://creativecommons.org/licenses/by-nc-nd/4.0/.

\section{References}

1. Chou PY, Hsieh YH, Lin CH. Necrotizing fasciitis of the entire head and neck: Literature review and case report. Biomed J 2020;43:94-8.

2. Sandner A, Moritz S, Unverzagt S, et al. Cervical Necrotizing Fasciitis--The Value of the Laboratory Risk Indicator for Necrotizing Fasciitis Score as an Indicative Parameter. J Oral Maxillofac Surg 2015;73:2319-33.

3. Paz Maya S, Dualde Beltran D, Lemercier P, et al. Necrotizing fasciitis: an urgent diagnosis. Skeletal Radiol 
2014;43:577-89.

4. Mtenga AA, Kalyanyama BM, Owibingire SS, et al. Cervicofacial necrotizing fasciitis among patients attending the Muhimbili National Hospital, Dar es Salaam, Tanzania. BMC Infect Dis 2019;19:642.

5. Taviloglu K, Cabioglu N, Cagatay A, et al. Idiopathic necrotizing fasciitis: risk factors and strategies for management. Am Surg 2005;71:315-20.

6. Thomas AJ, Mong S, Golub JS, et al. Klebsiella pneumoniae cervical necrotizing fasciitis originating as an abscess. Am J Otolaryngol 2012;33:764-6.

7. Baran J, Jr., Abdo WM, Merritt KW, et al. Tricuspid valve endocarditis due to a moderately susceptible Streptococcus constellatus: persistent bacteremia and fatal outcome despite penicillin plus gentamicin therapy. Scand J Infect Dis 1998;30:420-1.

Cite this article as: Jin L, Fan K, Liu S, Yu S. Necrotizing fasciitis of the jaw, neck and mediastinum caused by Klebsiella oxytoca and Streptococcus constellatus: a case report. Ann Palliat Med 2021;10(7):8431-8436. doi: 10.21037/apm-20-2427
8. Cheng NC, Tai HC, Chang SC, et al. Necrotizing fasciitis in patients with diabetes mellitus: clinical characteristics and risk factors for mortality. BMC Infect Dis 2015;15:417.

9. Petitpas F, Blancal JP, Mateo J, et al. Factors associated with the mediastinal spread of cervical necrotizing fasciitis. Ann Thorac Surg 2012;93:234-8.

10. Cruz Toro P, Callejo Castillo A, Tornero Salto J, et al. Cervical necrotizing fasciitis: report of 6 cases and review of literature. Eur Ann Otorhinolaryngol Head Neck Dis 2014;131:357-9.

11. Sarna T, Sengupta T, Miloro M, et al. Cervical necrotizing fasciitis with descending mediastinitis: literature review and case report. J Oral Maxillofac Surg 2012;70:1342-50.

12. Hanna BC, Delap TG, Scott K, et al Surgical debridement of craniocervical necrotizing fasciitis: the window of opportunity. J Laryngol Otol 2006;120:702-4. 